\title{
Fitting a Curve, Cutting Surface, and Adjusting the Shapes of Developable Hermite Patches
}

\author{
Kusno \\ Department of Mathematics, University of Jember, Indonesia \\ Received October 15, 2020; Revised November 17, 2020; Accepted December 20, 2020
}

\section{Cite This Paper in the following Citation Styles}

(a): [1] Kusno , "Fitting a Curve, Cutting Surface, and Adjusting the Shapes of Developable Hermite Patches," Mathematics and Statistics, Vol. 8, No. 6, pp. 740 - 746, 2020. DOI: 10.13189/ms.2020.080615.

(b): Kusno (2020). Fitting a Curve, Cutting Surface, and Adjusting the Shapes of Developable Hermite Patches. Mathematics and Statistics, 8(6), 740 - 746. DOI: 10.13189/ms.2020.080615.

Copyright $\odot 2020$ by authors, all rights reserved. Authors agree that this article remains permanently open access under the terms of the Creative Commons Attribution License 4.0 International License

\begin{abstract}
Formulation of developable patches is beneficial for modeling of the plate-metal sheet in the based-metal-industries objects. Meanwhile, installing the developable patches on a frame of the items and making a hole on these objects surface still need some practical techniques for developing. For these reasons, this research aims to introduce some methods for fitting a curve segment, cutting the developable patches, and adjusting their formulas. Using these methods can design various profile shapes of rubber filer installed on a frame of the objects and create a fissure or hole on the patches' surface. The steps are as follows. First, we define the planes containing the patches' generatrixes and orthogonal to the boundary curves. Then, it fits the Hermite and Bézier curve, via arranging some control points data on these planes, to model the rubber filler shapes. Second, we numerically evaluate a method for cutting the patches with a plane and adjusting the patches' form by modifying their formula from a linear interpolation form into a combination of curve and vectors forms. As a result, it can present some equations and procedures for plotting required curves, cutting surfaces, and modifying the extensible or narrowable shape of Hermite patches. These methods offer some advantages and contribute to designing the based-metal-sheets' object surfaces, especially modeling various forms of rubber filer profiles installed on a frame of the objects and making hole shapes on the plate-metal sheets.
\end{abstract}

Keywords Fitting, Cutting, Adjusting, Curves, Surfaces, Developable Hermite Patches

\section{Introduction}

Some developable surfaces' formulas can be used to model automobile parts, ship hulls, and aircraft [1,2,3,4]. Al-Ghefari and Abdel-Baky [5] presented the procedure to construct the developable surfaces and identify these surfaces into three types, i.e., a cylinder, cone, and tangent surface. Then, Xu et al. [6] discussed the minimal surface formulation via a given boundary curve of the surface. In this case, they use a quasi-harmonic Bézier approximation and a quasi-harmonic mask. After that, $\mathrm{Hu}$ et al. [7] formulated the developable Bézier-like surfaces with Bernstein-like basis functions. Kusno [8] discussed the construction of regular developable Bézier patches in which their boundary curves are defined by the combination of four, five, and six degrees. Then, he developed this method by applying Hermite polynomial curves [9]. Lately, Fernández and Pérez [10] introduced the technique for designing the developable surfaces using their boundary curves in the form of NURBS curves.

To model the surface parts of the based-metal-industries objects aided by the developable surfaces' formulas, it needs some practical calculations. For this reason, the paper presents a new approach for fitting, cutting, and modeling the parts of developable Hermite patches in the following steps of discussion. First, we review the numerical calculation of cubic and quintic Hermite developable patches introduced. Second, this study evaluates the method for fitting a curve segment and for modifying the developable Hermite patches. Third, we discuss a technique for cutting and amend the shapes of the pieces. Finally, the results of the study are summarized. 


\section{Formulation of Cubic and Quintic Hermite Developable Patches}

In this section, we study the developable condition of the Hermite developable patches supported by two parallel planes. Then, it reviews the construction of the cubic and quintic Hermite developable patches that were introduced.

Definition: The ruled surface $\mathbf{S}(u, v)=\mathbf{f}(u)+v \mathbf{g}(u)$ is developable, if the tangent plane is constant along each generatrix, that is the vectors $\left[\mathbf{g}^{\prime}(u), \mathbf{f}^{\prime}(u), \mathbf{g}(u)\right]$ are coplanar $[8,11]$.

Consider $\mathbf{g}(u)=\mathbf{q}(u)-\mathbf{P}(u)$ and $\mathbf{f}(u)=\mathbf{P}(u)$. It can state that

$$
\mathbf{S}(u, v)=\mathbf{f}(u)+v \cdot \mathbf{g}(u)=(1-v) \mathbf{P}(u)+v \cdot \mathbf{q}(u) .
$$

If the vectors $\left[\mathbf{g}^{\prime}(u), \mathbf{f} '(u), \mathbf{g}(u)\right]$ must be coplanar that can affirm the tangent vector $\mathbf{q}^{\prime}(u)=\tau(u) . \mathbf{P}^{\prime}(u)+\sigma(u)$. $[\mathbf{q}(u)-\mathbf{P}(u)]$ with $\tau(u)$ and $\sigma(u)$ two real scalars. In the application, we necessitate that the parametric functions $\mathbf{P}(u)$ and $\mathbf{q}(u)$ are respectively in the planes $\Psi_{1} / / \Psi_{2} / / Z O Y$ and It requires that $\tau(u)$ is positive constant $\tau$. Because of this reason, it can formulate the developable condition in the form $[1,8]$

$$
\mathbf{q}^{\prime}(u)=\tau . \mathbf{P}^{\prime}(u) .
$$

When the value $\tau=1$, the surface shape will be a cone in which their generatrixes meet at a point. Contrary, when the value $\tau \neq 1$, the surface will be a cylinder, and their generatrixes will be parallel. Consequently, for any two selected generatrices, it must be coplanar.

Consider a cubic polynomial Hermite curve $\mathbf{P}_{3}(u)=\mathbf{a}_{3} u^{3}$ $+\mathbf{b}_{3} u^{2}+\mathbf{c}_{3} u+\mathbf{d}_{3}$. We require at $\mathbf{P}_{3}(0)=\mathbf{a}_{3} \cdot 0^{3}+\mathbf{b}_{3} \cdot 0^{2}+\mathbf{c}_{3} .0$ $+\mathbf{d}_{3}=\mathbf{P}_{\mathrm{o}} ; \mathbf{P}_{3}(1)=\mathbf{a}_{3} \cdot 1^{3}+\mathbf{b}_{3} \cdot 1^{2}+\mathbf{c}_{3} \cdot 1+\mathbf{d}_{3}=\mathbf{P}_{1} ; \mathbf{P}_{3}(1 / 2)=$ $\mathbf{a}_{3} .(1 / 2)^{3}+\mathbf{b}_{3} .(1 / 2)^{2}+\mathbf{c}_{3} .(1 / 2)+\mathbf{d}_{3}=\mathbf{P}_{1 / 2}$, and the tangent vectors $\mathbf{P}_{3}^{u}(0)=3 \cdot \mathbf{a}_{3} \cdot 0^{2}+2 \cdot \mathbf{b}_{3} \cdot 0+\mathbf{c}_{3}=\mathbf{P}_{0}$ u. Therefore, the coefficients of the the Hermite curve $\mathbf{P}_{3}(\mathrm{u})$ are $\mathbf{a}_{3}=$ $2 \mathbf{P}_{1}-8 \mathbf{P}_{1 / 2}+2 \mathbf{P}_{o}^{u}+6 \mathbf{P}_{0} ; \mathbf{b}_{3}=8 \mathbf{P}_{1 / 2}-\mathbf{P}_{1}-3 \mathbf{P}_{o}^{u}-7 \mathbf{P}_{\mathrm{o}} ; \mathbf{c}_{3}=\mathbf{P}_{o}^{u} ; \mathbf{d}_{3}=\mathbf{P}_{0}$. As a result, it can formulate $\mathbf{P}_{3}(\mathrm{u})$ in the geometric representation

$$
\mathbf{P}_{3}(u)=H_{1}(u) \mathbf{P}_{\mathrm{o}}+H_{2}(u) \mathbf{P}_{1 / 2}+H_{3}(u) \mathbf{P}_{1}+H_{4}(u) \mathbf{P}_{o}^{u}
$$

with

$$
\begin{gathered}
H_{1}(u)=6 u^{3}-7 u^{2}+1 ; H_{2}(u)=-8 u^{3}+8 u^{2} ; \\
H_{3}(u)=2 u^{3}-u^{2} ; H_{4}(u)=2 u^{3}-3 u^{2}+u .
\end{gathered}
$$

Using the same calculation method of this cubic Hermite curve, the quintic Hermite curve $\mathbf{P}_{5 x}(u)$ constructed by the endpoints $\mathbf{P}_{5 x}(0)=\mathbf{P}_{0}, \mathbf{P}_{5 x}(1)=\mathbf{P}_{1}$, one intermediate point $\mathbf{P}_{5 x}(1 / 2)=\mathbf{P}_{1 / 2}$, and the tangent vectors $\mathbf{P}_{5 x}^{u}(0)=\mathbf{P}_{o}^{u}, \mathbf{P}_{5 x}^{u}(1 / 2)$ $=\mathbf{P}_{1 / 2}^{u}, \mathrm{P}_{5 \mathrm{x}}^{\mathrm{u}}(1)=\mathbf{P}_{1}^{u}$ is

$$
\begin{gathered}
\mathbf{P}_{5 x}(u)=H_{1}(u) \cdot \mathbf{P}_{\mathrm{o}}+H_{2}(u) \cdot \mathbf{P}_{1 / 2}+H_{3}(u) \cdot \mathbf{P}_{1}+H_{4}(u) \cdot \mathbf{P}_{o}^{u}+ \\
H_{5}(u) \cdot \mathbf{P}_{1 / 2}^{u}+H_{6}(u) \cdot \mathbf{P}_{1}^{u} .
\end{gathered}
$$

with

$$
\begin{gathered}
H_{1}(u)=24 u^{5}-68 u^{4}+66 u^{3}-23 u^{2}+1 ; \\
H_{2}(u)=16 u^{4}-32 u^{3}+16 u^{2} ;
\end{gathered}
$$

$$
\begin{gathered}
H_{3}(u)=-24 u^{5}+52 u^{4}-34 u^{3}+7 u^{2} ; \\
H_{4}(u)=4 u^{5}-12 u^{4}+13 u^{3}-6 u^{2}+u \\
H_{5}(u)=16 u^{5}-40 u^{4}+32 u^{3}-8 u^{2} \\
H_{6}(u)=4 u^{5}-8 u^{4}+5 u^{3}-u^{2} .
\end{gathered}
$$

In contrast, the formula of quintic polynomial Hermite curve $\mathbf{P}_{5 y}(u)$ determined by endpoints $\mathbf{P}_{5 y}(0)=\mathbf{P}_{0}, \mathbf{P}_{5 y}(1)=$ $\mathbf{P}_{1}$, two intermediate points $\mathbf{P}_{5 y}(1 / 3)=\mathbf{P}_{1 / 3}$ and $\mathbf{P}_{5 y}(2 / 3)=$ $\mathbf{P}_{2 / 3}$, two tangent vectors $\mathbf{P}_{5 y}^{u}(0)=\mathbf{P}_{o}^{u}$ and $\mathrm{P}_{5 \mathrm{y}}^{\mathrm{u}}(1)=\mathbf{P}_{1}^{u}$ is

$$
\begin{gathered}
\mathbf{P}_{5 y}(u)=H_{1}(u) \mathbf{P}_{\mathrm{o}}+H_{2}(u) \mathbf{P}_{1 / 3}+H_{3}(u) \mathbf{P}_{2 / 3}+H_{4}(u) \mathbf{P}_{1}+ \\
H_{5}(u) \mathbf{P}_{o}^{u}+H_{6}(u) \mathbf{P}_{1}^{u}
\end{gathered}
$$

with

$$
\begin{gathered}
H_{1}(u)=117 / 4 \cdot u^{5}-333 / 4 \cdot u^{4}+323 / 4 \cdot u^{3}-111 / 4 \cdot u^{2}+1 ; \\
H_{2}(u)=-243 / 4 \cdot u^{5}+162 \cdot u^{4}-567 / 4 \cdot u^{3}+81 / 2 \cdot u^{2} ; \\
H_{3}(u)=243 / 4 \cdot u^{5}-567 / 4 \cdot u^{4}+405 / 4 \cdot u^{3}-81 / 4 \cdot u^{2} ; \\
H_{4}(u)=-117 / 5 \cdot u^{5}+63 \cdot u^{4}-161 / 4 \cdot u^{3}+15 / 2 \cdot u^{2} ; \\
H_{5}(u)=9 / 2 \cdot u^{5}-27 / 2 \cdot u^{4}+29 / 2 \cdot u^{3}-13 / 2 \cdot u^{2}+u ; \\
H_{6}(u)=9 / 2 \cdot u^{5}-9 \cdot u^{4}+11 / 2 \cdot u^{3}-u^{2} .
\end{gathered}
$$

Given two Hermite curves in the form $\mathbf{P}(u)$ and $\mathbf{q}(u)$ of equations (3), (4), and (5). These curves are respectively laid in the plane $\Upsilon_{1}, \Upsilon_{2}$, and $\Upsilon_{1} / / \Upsilon_{2} / / Y O Z$. Based on these restrictions, we will review the construction method of the developable Hermite patches

$$
\mathbf{L}(u, v)=(1-v) \cdot \mathbf{P}(\mathrm{u})+v \cdot \mathbf{q}(\mathrm{u})
$$

and $u, v$ in interval $0 \leq u, v \leq 1$. In this case, it can summarize that the formulation steps of the patch design are as follows [9].

a. Case 1: Curves $\mathbf{P}(u)$ and $\mathbf{q}(u)$ cubic of Equation (3)

If $\mathbf{P}(u)$ and $\mathbf{q}(\mathrm{u})$ are of Equation (3), then the developable criteria (2) of both boundary curves will be in the equation $\mathbf{q}^{\prime}(u)=\tau(u) \cdot \mathbf{P}^{\prime}(u)$ or

$$
\begin{gathered}
H_{1}{ }^{u}(u) \mathbf{q}_{\mathrm{o}}+H_{2}{ }^{u}(u) \mathbf{q}_{1 / 2}+H_{3}{ }^{u}(u) \mathbf{q}_{1}+H_{4}{ }^{u}(u) \mathbf{q}_{o}^{u}=\tau\left[H_{1}{ }^{u}(u)\right. \\
\left.\mathbf{P}_{\mathrm{o}}+H_{2}{ }^{u}(u) \mathbf{P}_{1 / 2}+H_{3}{ }^{u}(u) \mathbf{P}_{1}+H_{4}{ }^{u}(u) \mathbf{P}_{o}^{u}\right] .
\end{gathered}
$$

This means that $\mathbf{q}_{\mathrm{o}}=\tau \mathbf{P}_{\mathrm{o}}, \mathbf{q}_{1 / 2}=\tau \mathbf{P}_{1 / 2}, \mathbf{q}_{1}=\tau \mathbf{P}_{1}, \mathbf{q}_{\mathrm{o}}^{\mathrm{u}}=\tau$ $\mathbf{P}_{o}^{u}$. Due to two genaratrixes $\mathbf{P}_{\mathrm{o}} \mathbf{q}_{\mathrm{o}}$ and $\mathbf{P}_{1} \mathbf{q}_{1}$ must be laid in the same plane, this developability conditions can be stated

$$
\left[\mathbf{q}_{\mathrm{o}} \mathbf{q}_{1}\right]=\tau \mathbf{P}_{\mathrm{o}} \mathbf{P}_{1} ;\left[\mathbf{q}_{\mathrm{o}} \mathbf{q}_{1 / 2}\right]=\tau\left[\mathbf{P}_{\mathrm{o}} \mathbf{P}_{1 / 2}\right] ; \mathbf{q}_{\mathrm{o}}^{\mathrm{u}}=\tau \mathbf{P}_{o}^{u} .
$$

b. Case 2: Curves $\mathbf{P}(\mathrm{u})$ and $\mathbf{q}(\mathrm{u})$ quintic of Equation (4)

If $\mathbf{P}(u)$ and $\mathbf{q}(u)$ are of Equation (4), then, using the same calculation method of the case 1 will find the developable criteria (2) in the form

$$
\begin{aligned}
& {\left[\mathbf{q}_{o} \mathbf{q}_{1}\right]=\tau\left[\mathbf{P}_{o} \mathbf{P}_{1}\right] ;\left[\mathbf{q}_{o} \mathbf{q}_{1 / 2}\right]=\tau\left[\mathbf{P}_{o} \mathbf{P}_{1 / 2}\right] ;} \\
& \mathbf{q}_{o}^{\mathrm{u}}=\tau . \mathbf{P}_{o}^{u} ; \mathbf{q}_{1 / 2}^{u}=\tau . \mathbf{P}_{1 / 2}^{u} ; \mathbf{q}_{o}^{\mathrm{u}}=\tau . \mathbf{P}_{o}^{u} .
\end{aligned}
$$

c. Case 3: Curves $\mathbf{P}(u)$ and $\mathbf{q}(u)$ quintic of Equation (5)

If $\mathrm{P}(\mathrm{u})$ and $\mathrm{q}(\mathrm{u})$ are of Equation (5), then the developable criteria (2) are in the formula 
$\left[\mathbf{q}_{o} \mathbf{q}_{1}\right]=\tau\left[\mathbf{P}_{o} \mathbf{P}_{1}\right] ;\left[\mathbf{q}_{o} \mathbf{q}_{1 / 3}\right]=\tau\left[\mathbf{P}_{o} \mathbf{P}_{1 / 3}\right] ; \quad\left[\mathbf{q}_{o} \mathbf{q}_{2 / 3}\right]=\tau$ $\left[\mathbf{P}_{o} \mathbf{P}_{2 / 3}\right] ; \mathrm{q}_{o}^{\mathrm{u}}=\tau . \mathbf{P}_{o}^{u} ; \mathrm{q}_{\mathrm{o}}^{\mathrm{u}}=\tau \cdot \mathrm{P}_{1}^{\mathrm{u}}$.

Via Equation (7), (8), and (9), we can, generally, construct the cubic and quintic Hermite patches by using the steps:

a. Determine the data $\mathbf{P}_{o}, \mathbf{P}_{1 / 2}, \mathbf{P}_{1 / 3}, \mathbf{P}_{2 / 3}, \mathbf{P}_{1}, \mathbf{q}_{o}, \mathbf{q}_{1 / 2}, \mathbf{q}_{1 / 3}$, $\mathbf{q}_{2 / 3}, \mathbf{q}_{1}, \mathbf{P}_{o}^{u}, \mathbf{P}_{1 / 2}^{u}, \mathbf{P}_{1}^{\mathrm{u}}$ that meet $\left[\mathbf{P}_{o} \mathbf{P}_{1}\right] / /\left[\mathbf{q}_{o} \mathbf{q}_{1}\right]$, $\left[\mathbf{P}_{o} \mathbf{P}_{1 / 2}\right] / /\left[\mathbf{q}_{o} \mathbf{q}_{1 / 2}\right]$, $\left[\mathbf{P}_{o} \mathbf{P}_{2 / 3}\right] / /\left[\mathbf{q}_{o} \mathbf{q}_{2 / 3}\right] ;$

$\left[\mathbf{P}_{o} \mathbf{P}_{1 / 3}\right] / /\left[\mathbf{q}_{o} \mathbf{q}_{1 / 3}\right]$,

b. Compute $\tau=\left|\mathbf{q}_{o} \mathbf{q}_{1}\right| /\left|\mathbf{P}_{o} \mathbf{P}_{1}\right|$ and calculate the vector tangents $\mathrm{q}_{\mathrm{o}}^{\mathrm{u}}=\tau \mathbf{P}_{o}^{u}, \mathbf{q}_{1 / 2}^{u}=\tau \mathbf{P}_{1 / 2}^{u}, \mathrm{q}_{1}^{\mathrm{u}}=\tau \mathrm{P}_{1}^{\mathrm{u}}$;

c. Substitute the data and calculated tangent vectors into Equation (3), (4), and (5) such that the developable Hermite patches $\mathbf{L}(u, v)$ of Equation (6) are formulated.

As an illustration, let the data $\mathbf{P}_{o}=\langle 10,-45,15\rangle, \mathbf{P}_{1 / 2}=$ $\langle 10,0,75 / 2\rangle$ and $\mathbf{P}_{1}=\langle 10,45,30\rangle, \mathbf{q}_{o}=\langle-20,-90,15\rangle$, $\mathbf{q}_{1 / 2}=\langle-20,-10,55\rangle$, and $\mathbf{q}_{1}=\langle-20,70,125 / 3\rangle$. It fulfills $\left[\mathbf{P}_{o} \mathbf{P}_{1}\right] / /\left[\mathbf{q}_{o} \mathbf{q}_{1}\right], \quad\left[\mathbf{P}_{o} \mathbf{P}_{1 / 2}\right] / /\left[\mathbf{q}_{o} \mathbf{q}_{1 / 2}\right]$, and $\tau=16 / 9$. The tangent vectors are elected $\mathbf{P}_{o}^{u}=\langle 0,70,-10\rangle$, and after calculating Equation (7), It obtains $q_{o}^{u}=\langle 0,1120 / 9$, -160/9>. From Equation (3), then can formulate the cubic
Hermite curves

$$
\begin{gathered}
\mathbf{P}_{3}(u)=H_{1}(u)<10,-45,15>+H_{2}(u)<10,0,75 / 2>+H_{3}(u) \\
<10,45,30>+H_{4}(u)<0,70,-10>; \\
\mathbf{q}_{3}(u)=H_{1}(u)<-20,-90,15>+H_{2}(u)<-20,-10,55>+H_{3}(u) \\
<-20,70,125 / 3>+H_{4}(u)<0,1120 / 9,-160 / 9>.
\end{gathered}
$$

Using Equation (6), it can formulate the cubic developable Hermite patch, as shown in Figure 1a. On the other hand, Figure $1 \mathrm{~b}$ presents a quintic developable Hermite patch with the boundary curves of Equation (5) and the data $\mathbf{P}_{o}=\langle 20,-60,10\rangle, \mathbf{P}_{1 / 3}=\langle 20,-70 / 3,25\rangle, \mathbf{P}_{2 / 3}=$ $\langle 20,40 / 3,22\rangle, \mathbf{P}_{1}=\langle 20,50,25\rangle, \mathbf{q}_{o}=\langle-20,-90,15\rangle, \mathbf{q}_{1}$ $=\langle-20,70,36.82\rangle, \mathbf{P}_{o}^{u}=\langle 0,90,90\rangle, \mathbf{P}_{1}^{u}=\langle 0,90,-100\rangle$, the calculated control points and tangent vectors $\mathbf{q}_{1 / 3}=\langle-20,-110 / 3,36,8\rangle, \quad \mathbf{q}_{2 / 3}=\langle-20, \quad 50 / 3,32.4\rangle$, $\mathbf{q}_{o}^{u}=\langle 0,131,131\rangle$, and $\mathbf{q}_{1}^{u}=\langle 0,131,-145\rangle$.

Based on these developable Hermite pieces' formulations, we will evaluate the curves fitted on the patches' boundary curves. Then, this study presents the technique for cutting the developable Hermite patches and adjusting their shapes by using the boundary curves' formulation. All figures in this paper are presented by utilizing the tool (software) Maple.

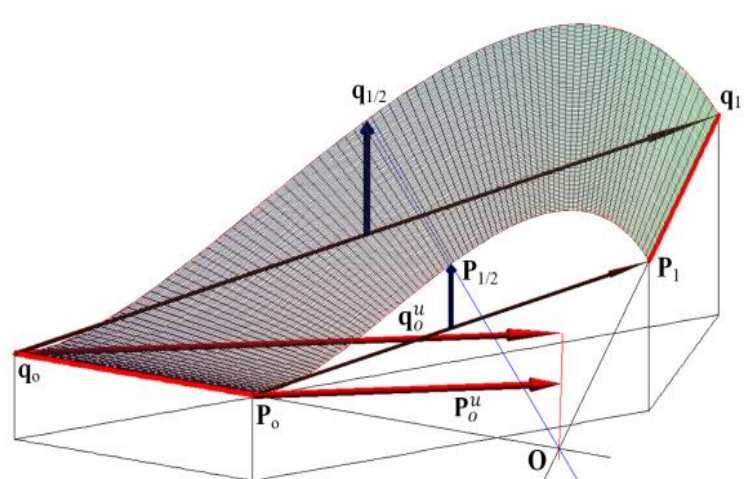

(a)

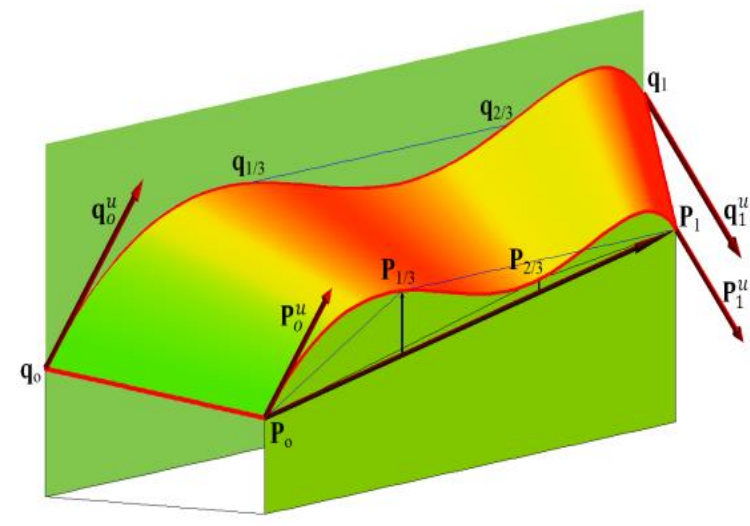

(b)

Figure 1. Cubic (a) and quintic (b) developable Hermite patches 


\section{Main Results}

\subsection{Fitting a Curve Segment on Developable Hermite Patches' Boundary Curves}

Given a real function of quintic polynomial $R_{5}(v)=a v^{5}$ $+b v^{4}+c v^{3}+d v^{2}+e v+f$ with the restrictions at $R_{5}(0)=$ $R_{\mathrm{o}}, R_{5}(1 / 5)=R_{1}, R_{5}(2 / 5)=R_{2}, R_{5}(3 / 5)=R_{3}, R_{5}(4 / 5)=R_{4}$ and $R_{5}(1)=R_{5}$. It can thus formulate the quintic Hermite polynomial curve $R_{5}(v)$ in this way

$$
\begin{aligned}
R_{5}(v)=N_{1}(v) R_{\mathrm{o}}+N_{2}(v) R_{1} & +N_{3}(v) R_{2}+N_{4}(v) R_{3}+N_{5}(v) R_{4} \\
+ & N_{6}(v) R_{5}
\end{aligned}
$$

with

$$
\begin{gathered}
N_{1}(v)=-625 / 24 v^{5}+625 / 8 v^{4}-125 / 24 v^{3}+375 / 8 v^{2}-37 / 12 v \\
+1 ; \\
N_{2}(v)=3125 / 24 v^{5}-4375 / 12 v^{4}+8875 / 24 v^{3}-1925 / 12 v^{2}+25 \\
v ; \\
N_{3}(v)=-3125 / 12 v^{5}+8125 / 12 v^{4}-7375 / 12 v^{3}+2675 / 12 \\
v^{2}-25 v ; \\
N_{4}(v)=3125 / 12 v^{5}+625 v^{4}+6125 / 12 v^{3}-325 / 2 v^{2}+50 / 3 v ; \\
N_{5}(v)=-3125 / 24 v^{5}+6875 / 24 v^{4}-5125 / 24 v^{3}+525 / 24 \\
v^{2}-25 / 4 v ; \\
N_{6}(v)=625 / 24 v^{5}-625 / 12 v^{4}+875 / 24 v^{3}-125 / 12 v^{2}+v .
\end{gathered}
$$

In another side, the quintic Bézier polynomial of the control points $B_{0}, B_{1}, B_{2}, B_{3}, B_{4}$, and $B_{5}$ is as follows

$$
\begin{gathered}
B_{5}(v)=B_{0}(1-v)^{5}+5 B_{1}(1-v)^{4} v+10 B_{2}(1-v)^{3} v^{2}+10 B_{3} \\
(1-v)^{2} v^{3}+5 B_{4}(1-v) v^{4}+B_{5} v^{5}
\end{gathered}
$$

with $0 \leq v \leq 1$.

Concerning the application of both equations (10) and (11), in this section, the study will introduce a new approach to formulate a fitting curve segment that can apply to design a model of a rubber filler along the borders $\mathrm{P}(\mathrm{u})$ and $\mathrm{q}(\mathrm{u})$ of the developable Hermite patches $\mathrm{L}(u, v)$ in Equation (6). It can also be used to set the installation shape of the developable patches $\mathrm{L}(u, v)$ on the planes $\Psi_{1} / / \Psi_{2} / /$ YOZ. The numerical solution method is as follows.

Consider the curve $\mathbf{P}(u)$ in the plane $\Psi_{1}, \mathbf{q}(u)$ in the plane $\Psi_{2}$. We define the unity vector $\mathbf{u}_{1}=[\mathbf{P}(u)-$ $\mathbf{q}(u)] /|\mathbf{P}(u)-\mathbf{q}(u)|$. Meanwhile, the unity tangent vector $\mathbf{t}(u)$ of the boundary curve $\mathbf{P}(u)$ is $\mathrm{t}(\mathrm{u})=$ $\mathrm{P}^{\mathrm{u}}(\mathrm{u}) /\left|\mathrm{P}^{\mathrm{u}}(\mathrm{u})\right|$ for $0 \leq u \leq 1$. Using both vectors $\mathbf{t}$ and $\mathbf{u}_{1}$, it can find a unity vector $\mathbf{u}_{2}=\mathbf{u}_{1} \wedge \mathbf{t}$. Based on these triple orthonormal unity vectors $\left[\mathbf{t}, \mathbf{u}_{1}, \mathbf{u}_{2}\right]$, we will draw and evaluate a polygon shape or a curve in the plane $\left[\mathbf{u}_{1}, \mathbf{u}_{2}\right]$ that can be moved (swabbed) orthogonally along the curve $\mathbf{P}(u)$ to model the cross-section profile curves of the rubber filler. For this purpose, it can arrange the control points' coordinate frame and apply the equations $(10,11)$ with steps in this way.

1. Calculate the vector $\mathbf{u}_{1}=[\mathbf{P}(u)-\mathbf{q}(u)] /$ $|\mathbf{P}(u)-\mathbf{q}(u)|=\left\langle u_{1 x}, u_{1 y}, u_{1 z}\right\rangle$ and the unity tangent vector $\mathbf{t}(u)=\mathbf{P}^{u}(u) /\left|\mathbf{P}^{u}(u)\right|=<\mathrm{t}_{1}, \mathrm{t}_{2}, \mathrm{t}_{3}>$, and $\quad \mathbf{u}_{2}=\mathbf{u}_{1} \wedge \mathbf{t}(u)=<u_{1 y} t_{3}-u_{1 z} t_{2}, u_{1 z} t_{1}-$ $u_{1 x} t_{3}, u_{1 x} t_{2}-u_{1 y} t_{1}>$ with $0 \leq u \leq 1$.

2. Set the position of control points coordinate $\left(0, R_{0}\right)$, $\left(0.2, R_{1}\right), \quad\left(0.4, R_{2}\right), \quad\left(0.6, R_{3}\right), \quad\left(0.8, R_{4}\right), \quad\left(1, R_{5}\right)$ for Equation $(10)$, and $\left(0, B_{0}\right),\left(0.2, B_{1}\right),\left(0.4, B_{2}\right),\left(0.6, B_{3}\right)$, $\left(0.8, B_{4}\right)$, and $\left(1, B_{5}\right)$ for Equation $(11)$ as shown in Figure 2a.

3. To design the cross-section profile of rubber filler in the plane $\left[\mathbf{u}_{1}, \mathbf{u}_{2}\right]$, compute the fitted curve shapes $\Gamma(v)$ $=[\alpha(v) . \mathbf{C}(v)]$ that are controlled by the real function $\alpha(v)$ of Equation (10) and (11) with their control points respectively in step (2) and the intermediate curve $\mathbf{C}(v)$ of circle $\mathbf{C}(v)=\cos (\varphi) \mathbf{u}_{1}+\sin (\varphi) \mathbf{u}_{2}$ with $0 \leq \varphi \leq 2 \pi v$ and $0 \leq v \leq 1$.

4. Move orthogonally the curve $\Gamma(v)$ along the boundary curve $\mathbf{P}(u)$ that can be formulated by using

a. the formulation of pipe in the form [12,13,14]

$$
\mathbf{F}(u, v)=\mathbf{P}(u)+\alpha(v) \cdot\left[\cos (\varphi) \mathbf{u}_{1}+\sin (\varphi) \mathbf{u}_{2}\right]
$$

with $0 \leq u, v \leq 1$ and $0 \leq \varphi \leq 2 \pi v$; or

b. the curve $\Gamma(v)$ of quintic Bézier polynomial curve with the control points $\mathbf{B}_{\mathrm{o}}=\mathbf{P}(\mathrm{u})+x_{\mathrm{o}}[\mathbf{q}(\mathrm{u})-\mathbf{P}(\mathrm{u})]$; $\mathbf{B}_{1}=\mathbf{P}(\mathrm{u})+x_{1}[\mathbf{q}(\mathrm{u})-\mathbf{P}(\mathrm{u})] ; \mathbf{B}_{2}=x_{2}\left[-\mathbf{u}_{1}+\mathbf{u}_{2}\right] ; \mathbf{B}_{3}$ $=x_{3} \mathbf{u}_{2} ; \mathbf{B}_{4}=x_{4}\left[\mathbf{u}_{1}+\mathbf{u}_{2}\right] ; \mathbf{B}_{5}=x_{5} \mathbf{u}_{1}$ and $\mathbf{F}(u, v)$ in the form

$$
\begin{gathered}
\mathbf{F}(u, v)=\mathbf{P}(u)+\left[\mathbf{B}_{\mathrm{o}}(1-v)^{5}+5 \mathbf{B}_{1}(1-v)^{4} v+10 \mathbf{B}_{2}(1-v)^{3} v^{2}+\right. \\
\left.10 \mathbf{B}_{3}(1-v)^{2} v^{3}+5 \mathbf{B}_{4}(1-v) v^{4}+\mathbf{B}_{5} v^{5}\right]
\end{gathered}
$$

with the scalars $x_{i}$ of real values for $i=1,2, \ldots, 5$ and $0 \leq$ $u, v \leq 1$.

Given the data of the cubic developable Hermite patches' construction in Figure 1a. We simulate this method as follows. Due to $\left.\mathbf{P}(u)=H_{1}(u)<10,-45,15\right\rangle+H_{2}(u)$ $\left.\langle 10,0,75 / 2\rangle+H_{3}(u)\langle 10,45,30\rangle+H_{4}(u)<0,70,-10\right\rangle$, it will find $\mathbf{P}^{u}(u)=\left\langle 10 H_{1}{ }^{u}(u)+10 H_{2}{ }^{u}(u)+10 H_{3}{ }^{u}(u)\right.$, $-45 H_{1}{ }^{u}(u)+45 H_{3}{ }^{u}(u)+70 H_{4}{ }^{u}(u), 15 H_{1}{ }^{u}(u)+75 / 2 H_{2}{ }^{u}(u)+$ $\left.30 H_{3}{ }^{u}(u)-10 H_{4}{ }^{u}(u)\right\rangle=\left\langle x_{u}, y_{u}, z_{u}\right\rangle$ and the unity tangent vector $\mathbf{t}(u)=<x_{u}, y_{u}, z_{u}>/\left|<x_{u}, y_{u}, z_{u}>\right|=<$ $t_{1}, t_{2}, t_{3}>$. The vector $\mathbf{u}_{1}=[\mathbf{P}(u)-\mathbf{q}(u)] /|\mathbf{P}(u)-\mathbf{q}(u)|$ $=\left\langle u_{1 x}, u_{1 y}, u_{1 z}\right\rangle$ and $\mathbf{u}_{2}=<u_{1 y} t_{3}-u_{1 z} t_{2}, u_{1 z} t_{1}-$ $u_{1 x} t_{3}, u_{1 x} t_{2}-u_{1 y} t_{1}>$ with $0 \leq u \leq 1$. If we elect the control points for Equations (10) of the values $R_{\mathrm{o}}=2, R_{1}=2, R_{2}=3$, $R_{3}=3, R_{4}=2, R_{5}=10$, and for Equation (11) of the values $B_{\mathrm{o}}=2, B_{1}=3, B_{2}=1, B_{3}=2, B_{4}=0, B_{5}=5$, then, in the plane $\left[\mathbf{u}_{1}, \mathbf{u}_{2}\right]$, it will respectively obtain the cross-section curves $\Gamma_{R}(v)$ and $\Gamma_{B}(v)$ as shown in Figure 2a. Hereafter, using Equation (12) can find the model of the rubber filler' cross-section profile $\mathbf{F}_{R}(u, v)$ and $\mathbf{F}_{B}(u, v)$ as simulated in Figure $2 \mathrm{~b}$. If the developable patches $\mathbf{L}(u, v)$ are defined by $\mathbf{P}(u)$ and $\mathbf{q}(u)$ of quintic Hermite curves with the data of Figure $1 \mathrm{~b}$, and the calculated profile $\mathbf{F}_{B}(u, v)$, then it can draw the rubber filler profile as presented in Figure $2 \mathrm{c}$. On the other hand, applying Equation (13) with the fixed scalars values $x_{0}=0.2, x_{1}=0.1, x_{2}=5, x_{3}=10, x_{4}=5$, and $x_{5}=$ 4 will construct a rubber filler' profile shape $\mathbf{F}(u, v)$ as illustrated in Figure 2d. 


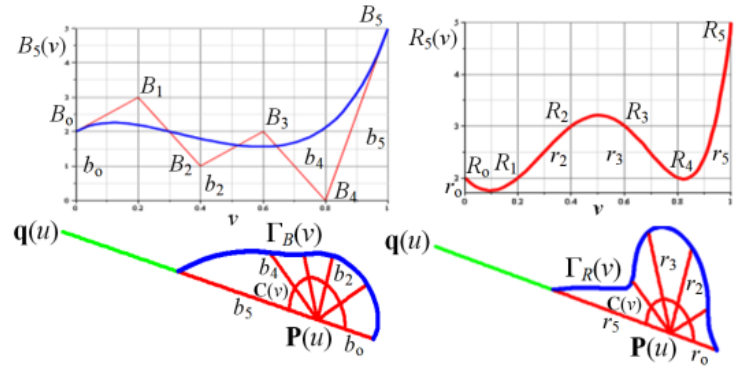

(a)

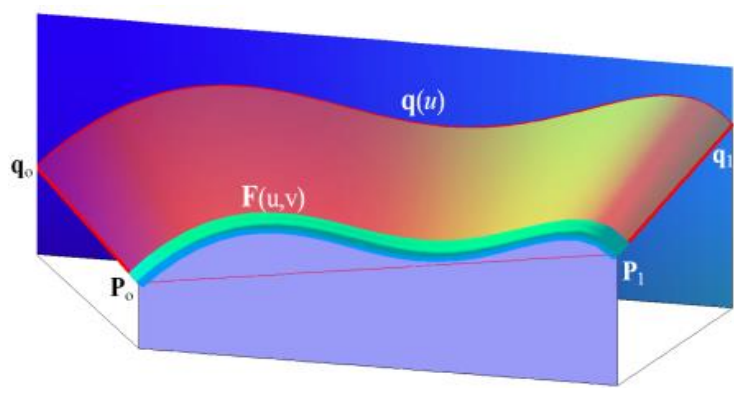

(c)

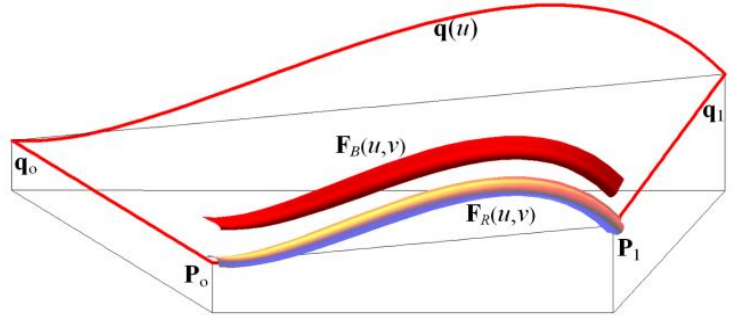

(b)

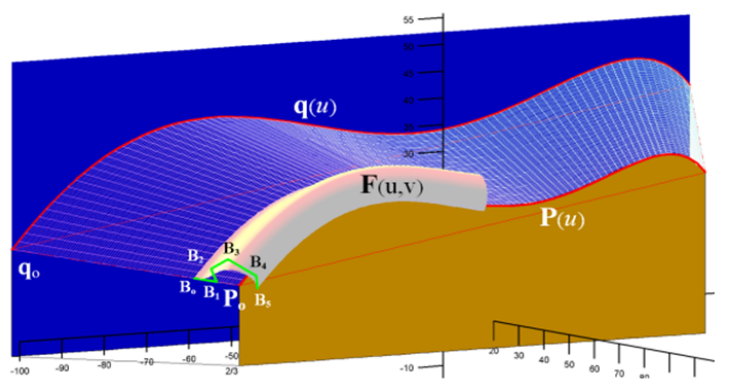

(d)

Figure 2. Designing profile shape of rubber filler

\subsection{Cutting Surface and Adjusting the Shapes of Developable Hermite Patches}

Consider a developable Hermite patch $\mathbf{L}(u, v)$ of Equation (6) with the endpoints of their boundary curves $\left[\mathbf{P}_{\mathrm{o}}, \mathbf{P}_{1}\right]$ and $\left[\mathbf{q}_{\mathrm{o}}, \mathbf{q}_{1}\right]$ in the plane $\Psi_{1} / / \Psi_{2} / / Y O Z$, respectively. We determine two alternative points $\mathbf{R}=(1-x) \mathbf{P}_{\mathrm{o}}+x \mathbf{q}_{\mathrm{o}}$ and $\mathbf{S}=(1-y) \mathbf{P}_{1}+y \mathbf{q}_{1}$ with $0 \leq x, y \leq 1$, then define a plane $\mathbf{T}(s, t)$ that passes to the line RS and perpendicular to the plane $\left[\mathbf{P}_{\mathrm{o}} \mathbf{P}_{1}, \mathbf{q}_{\mathrm{o}} \mathbf{q}_{1}\right]$ as shown in Figure $3 \mathrm{a}$. Due to the plane $\mathbf{T}(s, t)$ cuts the developable patch $\mathbf{L}(u, v)$, the problem that will be discussed is to calculate the surface part of the patch that is limited by the plane $\mathbf{T}(s, t)$ and $\Upsilon_{1}$. The solution method is as follows.

If the vector $\mathbf{n}=\left(\mathbf{P}_{\mathrm{o}} \mathbf{P}_{1}\right) \wedge\left(\mathbf{P}_{\mathrm{o}} \mathbf{q}_{\mathrm{o}}\right)$ is a normal vector of the plane $\left[\mathbf{P}_{\mathrm{o}} \mathbf{P}_{1}, \mathbf{q}_{\mathrm{o}} \mathbf{q}_{1}\right]$, then it can formulate the plane $\mathbf{T}(s, t)$ in the form

$$
\mathbf{T}(s, t)=\mathbf{R}+s .(\mathbf{R S})+\alpha . t . \mathbf{n}
$$

with $0 \leq s, t \leq 1$ and $\alpha$ real scalar. Because of the formulation $\mathbf{L}(u, v)=(1-v) \cdot \mathbf{P}(u)+v \cdot \mathbf{q}(u)=\mathbf{P}(u)+v \cdot[\mathbf{q}(u)-\mathbf{P}(u)]$, this problem means that, numerically, how can define the developable patch that is constructed by the generatrix lines $\mathbf{D}\left(u_{i}, v\right)=\mathbf{P}\left(u_{\mathrm{i}}\right)+v\left[\mathbf{q}\left(u_{\mathrm{i}}\right)-\mathbf{P}\left(u_{\mathrm{i}}\right)\right]$ and limited by the plane $\mathbf{T}(s, t)$ and $\Upsilon_{1}$ for the value $u_{i}=i / n$ and $i=0,1,2,3 \ldots, n$. These lines $\mathbf{D}\left(u_{i}, v\right)$ will design the developable Hermite strip patch. When $\mathbf{T}(s, t)$ and $\mathbf{D}\left(u_{i}, v\right)$ intersect, they must meet $\mathbf{T}(s, t)=\mathbf{D}\left(u_{i}, v\right)$ or

$$
\mathbf{R}+s .(\mathbf{R S})+t(\alpha . \mathbf{n})=\mathbf{P}\left(\mathbf{u}_{i}\right)+v\left[\mathbf{q}\left(\boldsymbol{u}_{i}\right)-\mathbf{P}\left(\boldsymbol{u}_{i}\right)\right] .
$$

Using the dot and cross multiplication of the vector algebra operations [11,15], for each value ui, it can, therefore, compute the parameter values

$$
\begin{gathered}
v_{\mathrm{i}}=\left[\left(\mathbf{R}-\mathbf{P}\left(u_{i}\right)\right) .(\mathbf{R S} \wedge(\alpha . \mathbf{n}))\right] /\left[\left(\mathbf{q}\left(u_{i}\right)-\right.\right. \\
\left.\left.-\mathbf{P}\left(u_{i}\right)\right) .(\mathbf{R S} \wedge(\alpha . \mathbf{n}))\right]
\end{gathered}
$$

for $i=0,1,2,3 \ldots, n$. Thus, to construct the surface part of developable Hermite strip patch $\mathbf{D}\left(u_{i}, v\right)$ bounded by the plane $\mathbf{T}(s, t)$ and $\Upsilon_{1}$, for each value $u_{i}$ and $i=0,1,2,3 \ldots, n$, it must define the parameter value $v$ in interval $0 \leq v \leq v_{i}$.

Figure 3a present the developable Hermite patch $\mathbf{L}(u, v)$ of Equation (6) and (4) by using the data $\left.\mathbf{R}=<-6,-84,14\rangle, \quad \mathbf{S}=<36,54,27,4\rangle, \quad \mathbf{P}_{o}=<50,-60,10\right\rangle$, $\left.\mathbf{P}_{1 / 2}=\langle 50,-5,27.5\rangle, \quad \mathbf{P}_{1}=\langle 50,50,25\rangle, \quad \mathbf{q}_{o}=<-20,-90,15\right\rangle$, $\mathbf{q}_{1}=\langle-20,70,36.82\rangle, \quad \mathbf{P}_{o}^{u}=\langle 0,30,90\rangle, \quad \mathbf{P}_{1 / 2}^{u}=$ $\langle 0,45,45\rangle, \mathbf{P}_{1}^{u}=\langle 0,90,-100\rangle$, the calculated control points and the tangent vector $\mathbf{q}_{1 / 2}=<-20,-10,40.9>, \mathbf{q}_{o}^{u}=$ $<0,45,135>, \mathbf{q}_{1 / 2}^{u}=\langle 0,68,68\rangle$,

$\mathbf{q}_{1}^{u}=\langle 0,135,-150\rangle$. The plane $\mathbf{T}$ is formulated $\mathbf{T}(s, t)=$ $\langle-6,-84,14\rangle+s .\langle 42,138,13.4\rangle+t .\langle 1.8,-1.9,13.9\rangle$ with $0 \leq s, t \leq 1$. As a result of the cutting $\mathbf{L}(u, v)$ with the plane $\mathbf{T}(s, t)$, it is shown in the red color of this figure $3 \mathrm{a}$. On the other hand, Figure $3 \mathrm{~b}$ illustrates the cutting $\mathbf{L}(u, v)$ by using the plan $\mathbf{T}(s, t)$ with the point positions $\mathbf{R}=\langle 36,-66,11\rangle, \mathbf{S}$ $=\langle-6,66,34.5\rangle$. When the plan $\mathbf{T}$ is oblique to the plane $\left[\mathbf{P}_{\mathrm{o}} \mathbf{P}_{1} \mathbf{q}_{1} \mathbf{q}_{\mathrm{o}}\right]$, we can also use Equation (16) to calculate the intersection between this plane $\mathbf{T}$ and the developable Hermite patch $\mathbf{L}(u, v)$. 


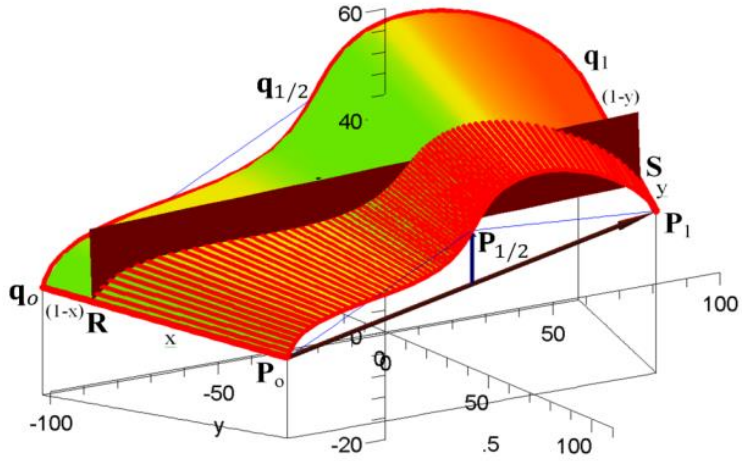

(a)

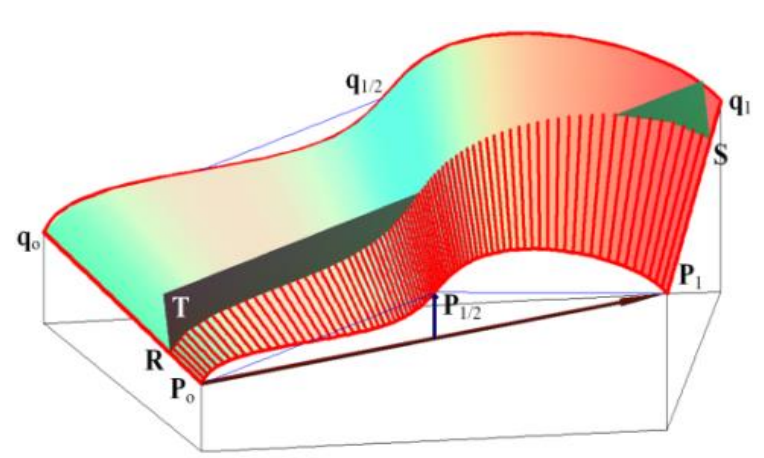

(b)

Figure 3. Cutting the developable Hermite patches using a plane $\mathbf{T}$ perpendicular to the plane $\left[\mathbf{P}_{0} \mathbf{P}_{1} \mathbf{q}_{1} \mathbf{q}_{\mathrm{o}}\right]$

In industrial applications, the surface form of the developable patches in Equation (6) sometimes needs to be modified in shape or measure. This surface area sometimes goes beyond the boundary curve, or even it must lay in the interior between both boundary curves of the patches. For this reason, based on their boundary curves $\mathbf{P}(u)$ and $\mathbf{q}(u)$, we adapt Equation (6) in the form of scalar and vector multiplication without changing their nature of developability. The technique is in this manner.

Consider the developable Hermite patch $\mathbf{L}(u, v)=(1-v)$ $\mathbf{P}(u)+v \mathbf{q}(u)=\mathbf{P}(u)+v[\mathbf{q}(u)-\mathbf{P}(u)]$. In this case, along the curve $\mathbf{P}(u)$, we want to control the measure of the generatrix lines $[\mathbf{q}(u)-\mathbf{P}(u)]$ such that the piece $\mathbf{L}(u, v)$ can be changed its surface profile. In other words, in the longitudinal direction $\mathbf{P}(u)$, it needs a real function' scalar $\sigma(u)$ so that, for $u$ defined in interval $0 \leq u \leq 1$, the vectors $\sigma(u)$. $[\mathbf{q}(u)-\mathbf{P}(u)]$ can be adjusted and arranged their measure and shape for design needs. So it replaces Equation (6) to become

$$
\mathbf{L}_{1}(u, v)=\mathbf{P}(u)+v \cdot \sigma(u) \cdot[\mathbf{q}(u)-\mathbf{P}(u)]
$$

$$
\mathbf{L}_{2}(u, v)=(1-v) .[\mathbf{P}(u)+\sigma(u) .(\mathbf{q}(u)-\mathbf{P}(u))]+v \mathbf{q}(u) .
$$

In this case, the scalar function $\sigma(u)$ can be elected as the real functions of quintic Hermite polynomial and quintic Bezier that are formulated in Equation (10), and (11) or of a trigonometric function. Let data $\mathbf{P}_{\mathrm{o}}=\langle 20,-60,10\rangle, \mathbf{P}_{1}=$ $\langle 20,50,25\rangle, \quad \mathbf{P}_{1 / 3}=\langle 20,-16,21\rangle, \quad \mathbf{P}_{2 / 3}=\langle 20,6,9\rangle, \quad \mathbf{P}_{o}^{u}=$ $\langle 0,90,-90\rangle, \quad \mathbf{P}_{1}^{u}=\langle 0,90,-80\rangle, \quad \mathbf{q}_{0}=\langle-20,-90,15\rangle, \quad \mathbf{q}_{1}=$ $\left\langle-20,70,36.8>\right.$. Via Equation (9), It can find $\mathbf{q}_{1 / 3}=$ $\langle-20,-26,31\rangle, \mathbf{q}_{2 / 3}=\langle-20,6,13.5\rangle, \mathbf{q}_{o}^{u}=\langle 0,131,-131\rangle, \mathrm{q}_{1}^{\mathrm{u}}$ $=\langle 0,131,-116,4\rangle$. If we elect $\sigma(u)$ of Equation (10) with $R_{\mathrm{o}}=0.4, R_{1}=0.5, R_{2}=0.6, R_{3}=0.3, R_{4}=0.2$ and $R_{5}=0.2$, then via Equation (17), obtain Figure 4a, but, when using Equation (18) will get Figure 4b. In Figure 4c,d, it shows five developable Hermite patches that can be defined by both Equation (17), (18) and the change of their shapes by moving the control point $\mathbf{P}_{1 / 2}$.
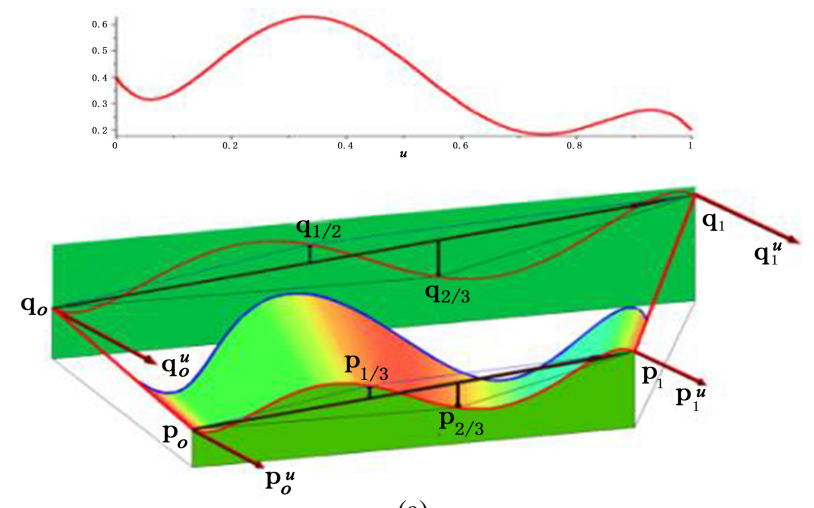

(a)
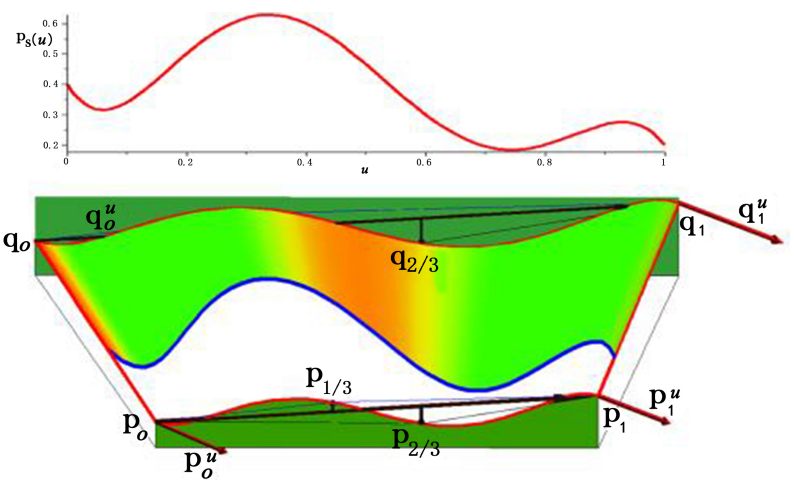

(b)

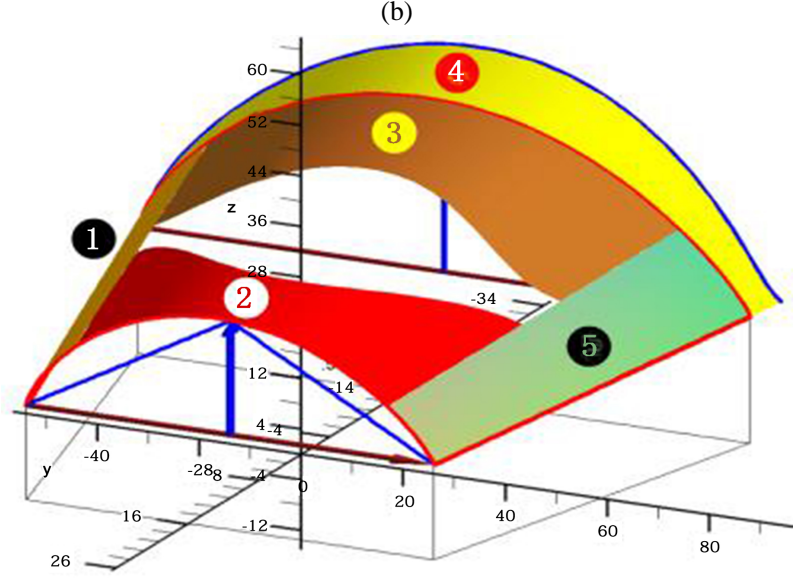

(c) 


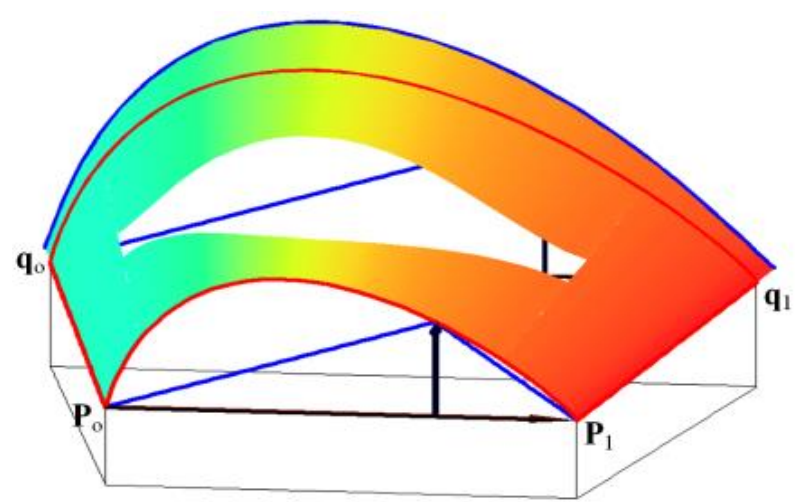

(d)

Figure 4. Shape adjustment of developable patches

\section{Conclusions}

Using the presented method of fitting a curve segment on developable Hermite patches' boundary curves can design the various rubber filler's cross-section profile curves. The process of cutting and adjusting the developable patches' shapes that were introduced will offer some advantages to model the surface parts of the based-metal-industries objects, for example, in making a hole on the plates or modifying the form of plate sheets. Hereafter, the exciting thing to develop is how to model the developable surfaces when their boundary curves are not laid in the planes.

\section{REFERENCES}

[1] W. H. Frey, D. Bindschadler. Computer-aided Design of a Class of Developable Bézier Surfaces, USA: R and D Publication 8057 General Motors, 1993.

[2] J. S. Chalfant. Analysis and Design of Developable Surfaces for Shipbuilding, These, Massachusetts Institute of Technology, USA, 1997.
[3] G. Elber. Approximation Algorithm for Developable Surfaces, CAGD, Vol.16, 539-556, 1995.

[4] G. Hu, J. L. Wu, X. Q. Qin. A New Approach in Designing of Local Controlled Developable H-Bézier Surfaces, Adv. Eng. Softw., Vol. 121, 26-38, 2018.

[5] R. A. Al-Ghefari, R. A. Abdel-Baky. An Approach for Designing Developable Surface with a Common Geodesic Curve, Int. J. Contemp. Math. Sci., Vol. 8, 875891, 2013.

[6] G. Xu, T. Rabczuk, E. Güler, Q. Wu, K. Hui, K. Wang. Quasi-harmonic Bézier Approximation of Minimal Surfaces for Finding Forms of Structural Membranes, Comput. Struct., Vol. 16, 55-63, 2015.

[7] G. Hu, H. X. Cao, S. X. Zhang, W. Guo. Developable Bézier-like Surfaces with Multiple Shape Parameters and Its Continuity Conditions, Appl. Math. Model., Vol. 45, 728$747,2017$.

[8] Kusno. Construction of Regular Developable Bézier Patches, Math. Comput. Appl., Vol. 24, No. 4, 1-13, 2019.

[9] Kusno. Modeling of Developable Surfaces Using Hermite Spline Interpolation Curves, Adv. Math.: Sci. J., Vol. 9, No. 10, 8431-8442, 2020.

[10] L. Fernández-Jambrina, F. Pérez-Arribas. Developable Surface Patches Bounded by NURBS Curves, J. Comp. Math., Vol. 38, 715-731, 2020.

[11] M. Lipschultz. Theory and Problems of Differential Geometry, Schaum's Outline Series, McGraw-Hill, New York, 1969.

[12] Kusno, A. C. Prihandoko. Continuous connection of two adjacent pipe parts defined by line, Bézier and Hermit center curves, J. Phys. Conf. Ser., Vol. 1008, No. 1, 1-7, 2018.

[13] Kusno. On the modeling of cross-section and longitudinal section of pipes, J. Phys. Conf. Ser., Vol. 1321, No. 2, 1-8, 2019.

[14] Kusno. Modeling pipes using pipes' center curves of quadratic and cubic spline interpolation, in Proceding of International Conference on Mathematics, Computational Sciences and Statistics (ICoMCoS 2020), AIP Conference Proceding 2020.

[15] M. E. Mortenson. Geometry Modeling, Jonh Wiley and Sons Inc., New York, 1996. 